\title{
Unblocking as a function of shock reduction
}

\author{
BARBARA H. BASDEN and DAVID M. KLEIM \\ California State University, Fresno, California 93740
}

\begin{abstract}
Contrasting predictions from Mackintosh and Turner's (1971) selective attention account and Rescorla and Wagner's (1972) nonselective account of unblocking were tested within a CER procedure employing UCS magnitude reduction. Magnitude of the UCS remained constant throughout all training stages for a blocking control group. The associative strength of a CS (light) introduced concurrently with UCS reduction was assessed in the third training stage. In that stage, reinforced presentations of tone (a "neutral" stimulus) were randomly intermingled with nonreinforced presentations of the tone-light compound. Rate oi acquisition of inhibition to the compound did not differ significantly for the two groups, a result which was discussed in terms of Mackintosh's (1975) revision of attention theory. Suppression to tone was significantly greater in the unblocking group than in the blocking control group, a result which calls into question the adequacy of CER designs in which shock intensity is varied.
\end{abstract}

Recent studies investigating the issue of selective vs. nonselective learning have frequently employed the conditioned emotional response (CER) procedure in which suppression of ongoing barpressing in the presence of a CS paired with shock is the dependent variable. Within this procedure, an excitatory CS is one which increases suppression, while an inhibitory CS is one which decreases suppression. The phenomenon of blocking in this procedure, absence of conditioning to an added CS (Cue B) following prior conditioning with another CS (Cue A), has been accounted for either in terms of selective attention to Cue A (e.g., Sutherland \& Mackintosh, 1971), or in terms of predictability of the UCS from the presence of A alone (Kamin, 1969; Rescorla \& Wagner, 1972).

Also subject to both interpretations is the associated phenomenon of unblocking, reinstatement of conditioning to the added CS when its introduction is accompanied by an alteration in UCS magnitude. When this alteration consists of UCS elimination, the added CS becomes inhibitory, as in unblocking studies reported by Kamin (1969) and by Mackintosh and Turner (1971). In terms of the Rescorla-Wagner model (1972), this follows from the assumption that the individual cues present on a conditioning trial are altered in associative strength as a function of the associative strength of the aggregate. Upon UCS elimination, the aggregate of A and $B$ would decrease in associative strength, but, since the associative strength of Cue $B\left(V_{B}\right)$ is zero upon its introduction, a decrease in $V_{B}$ would render it negative and, hence, inhibitory. Similarly, the model allows the prediction that $B$ would become inhibitory when UCS magnitude is reduced rather than eliminated. This

Requests for reprints should be sent to Barbara $\mathrm{H}$. Basden, Department of Psychology, California State University, Fresno 93740. The second author is now at the Department of Psychology, University of Missouri, Columbia 65201. This paper is sponsored by David R. Basden, who takes full editorial responsibility for its contents. assumes that the associative strength of $A\left(V_{A}\right)$ exceeds asymptotic conditioning supportable by the lower UCS intensity.

According to the attentional account of unblocking (Mackintosh \& Turner, 1971), the change in UCS magnitude which accompanies introduction of $B$ enables conditioning to that $\mathrm{CS}$, in that it is no longer redundant with A. Presumably, UCS elimination allows B to function as a "safe" signal, thus rendering it inhibitory. Extension of this analysis to a situation in which UCS magnitude is reduced rather than eliminated leads to the opposite prediction. If the subject attends to $B$ following alteration in UCS magnitude, that subject would learn that $B$ is accompanied by shock and B should become excitatory. The purpose of the present study was to provide a test of these diverging predictions in an unblocking study.

To allow assessment of the nature of the associative strength of $B$, a savings test was incorporated into the design. This consisted of the administration of reinforced trials with a neutral CS, Cue X, randomly intermingled with nonreinforced trials with the compound CS, BX. Presence of either excitatory or inhibitory strength for $B$ was assessed against the acquisition of suppression to $\mathrm{X}$ alone.

\section{METHOD}

\section{Subjects and Apparatus}

The subjects were 16 naive male Sprague-Dawley rats maintained at $80 \%$ of free-feeding weight. They were approximately 70 days old at the beginning of the experiment.

The apparatus consisted of a Scientific Prototype rodent test chamber inside a sound-attenuating enclosure, with inside demensions of $81 \times 44.5 \times 40.6 \mathrm{~cm}$. Speakers mounted on the front and rear walls of the enclosure were employed to present white noise $(\mathrm{N})$ and a $1,000-\mathrm{Hz}$ tone $(\mathrm{T})$, respectively. Two high-intensity lamp bulbs were mounted in the ceiling of the enclosure for presentation of the light cue (L). This light was diffused through a translucent plastic covering affixed to the lid of the chamber. Automatic programming equipment was located outside the enclosure. 


\section{Procedure}

During pretraining, the subjects were first shaped to barpress and then given FR 2, FR 5, FR 10, and, finally, VI 30-sec schedules on successive days. The VI 30 -sec schedule was maintained throughout the experiment, with CER training instituted when a steady rate of responding was first obtained on this schedule. Following pretraining, subjects were randomly assigned to unblocking (Group U) or blocking (Group B) conditions.

Each daily training session was $60 \mathrm{~min}$ in length, with four conditioning trials per session. Throughout all training stages, the CS was presented for $60 \mathrm{sec}$, followed immediately (on reinforced trials) by a 1-sec footshock UCS. Presentation times were randomized within and between sessions, with the restriction that no presentations were given within $5 \mathrm{~min}$ from the beginning or end of a session and presentations were separated by at least $7 \mathrm{~min}$. Stage 1 consisted of five sessions (20 reinforced trials) with $\mathrm{N}$; Stage 2 consisted of four sessions (16 reinforced trials) with the compound NL; and Stage 3 consisted of six sessions, with each comprised of one reinforced trial with $T$ and three nonreinforced trials with the compound TL (a total of 6 reinforced T trials and 18 nonreinforced TL trials). The two groups differed in that, for Group U, the UCS intensity was $5 \mathrm{~mA}$ during Stage 1 and $1 \mathrm{~mA}$ during Stages 2 and 3; whereas, for Group B, UCS intensity was $1 \mathrm{~mA}$ throughout all three training stages. Number of barpresses was recorded during CS presentation (D) and during the $60 \mathrm{sec}$ preceding CS presentation (P); the dependent variable employed was a suppression ratio defined as $\mathrm{D} /(\mathrm{P}+\mathrm{D})$.

\section{RESULTS AND DISCUSSION}

For the final session of Stage 1 , the overall mean suppression ratio to $\mathrm{N}$ was .13 for Group $\mathrm{B}$ and .05 for Group $\mathrm{U}$, Mann-Whitney $\mathrm{U}(8,8)=8.5, \mathrm{p}<.05$. Thus, suppression was greater in Group $U$ than in Group B, a result which is likely to occur with the more intense UCS. For the initial session of Stage 2, the overall mean suppression ratios to the NL compound did not differ for the two groups, $\mathrm{U}(8,8)=30.5, \mathrm{p}>.10$, with suppression essentially complete for all subjects. Overall mean suppression ratios were .05 in Group $U$ and .06 in Group B.

Mean suppression ratios to $\mathrm{T}$ on the initial trial of Stage 3 were .02 and .20 in Groups $U$ and B, respectively, $\mathrm{U}(8,8)=8.5, \mathrm{p}<.05$. Thus, although neither group had experienced $\mathrm{T}$ previously, initial suppression to it was greater in Group U. In our opinion, this initial difference can be attributed to the more intense UCS employed in Stage 1 for Group U. Groups U and B did not differ at the conclusion of Stage 2 training; suppression to the NL compound was essentially perfect

Table 1

Mean Suppression Ratios to Both CSs During Stage 3

\begin{tabular}{lcccccc}
\hline & \multicolumn{6}{c}{ Sessions } \\
& 1 & 2 & 3 & 4 & 5 & 6 \\
\hline Group B & & & & & & \\
T & .27 & .24 & .18 & .18 & .11 & .15 \\
TL & .16 & .20 & .25 & .23 & .32 & .38 \\
Group U & & & & & & \\
$\underset{T}{T}$ & .10 & .08 & .02 & .05 & .05 & .02 \\
TL & .07 & .11 & .09 & .13 & .22 & .23 \\
\hline
\end{tabular}

for both groups. Thus, the basis for differential suppression to $\mathrm{T}$ must be attributed to differential excitatory tendencies established in Stage 1. This implies that the effect of experiencing a given shock intensity in one context is not limited to that context but can also alter excitatory tendencies in the presence of "neutral" stimuli. Thus, such complex testing procedures as those employed in the current study and in the Mackintosh and Turner study (1971) are open to interpretational difficulties stemming from a subject's history with differing shock intensities.

Mean suppression ratios to TL on the initial trial with that compound were .09 and .15 for Groups $U$ and B, respectively, $U(8,8)=29, p>.10$, indicating no initial Stage 3 differences. Thus, although $\mathrm{T}$ was an excitatory stimulus, $L$ was no more excitatory for subjects in Group U than for subjects in Group B. These results thus do not support the attentional explanation (Mackintosh \& Turner, 1971) of the unblocking phenomenon. Mean suppression ratios for each of the six sessions in Stage 3 are shown in Table 1.

To determine if $\mathrm{L}$ was initially inhibitory, a 2 (Groups) by 2 (CS Type) analysis of variance was conducted on the mean suppression ratios for the first two sessions of Stage 3. The T and TL scores were obtained by averaging the respective suppression ratios. Neither the main effect of Groups, $F(1,14)=2.20, p>.10$, nor of CS Type, $F(1,14)=1.67, \mathrm{p}>.10$, was significant; however, the interaction of these two variables was significant, $F(1,14)=13.13, p<.01$. Tests of simple main effects indicated that suppression to TL and to T did not differ for Group $U, F<1$, but was greater to TL than to $T$ for Group $B, F(1,7)=6.75, p<.05$. These results indicate that initially $\mathrm{L}$ was not an inhibitory stimulus for Group B. This failure to obtain blocking was not totally unexpected in that Kamin (1969) has previously reported that more sensitive measures, such as the relearning measure employed here, do reveal some conditioning to the redundant $\mathrm{CS}$ in blocking designs.

An analysis of all six sessions in Stage 3 indicated that suppression was significantly greater in Group $U$ than in Group B, $F(1,14)=4.57, p<.05$, suppression to $T$ was greater than to $T L, F(1,14)=9.56, p<.01$, and that the difference between suppression ratios to $\mathrm{T}$ and $\mathrm{TL}$ increased with sessions, $F(5,70)=16.01, p<.01$. No other factors in this analysis were significant, including the Groups by CS Type by Sessions interaction, $F(5,70)$ $=1.25, \mathrm{p}>.10$. This interaction is of some theoretical importance in that modified attention theory (Mackintosh, 1975) would lead to the prediction that L would more rapidly become an inhibitory stimulus for subjects in Group U than for subjects in Group B. This assumes that the learning rate $(\alpha)$ for the added cue, B, in a blocking design, would be reduced if introduction of that cue is not accompanied by a change in the UCS. Thus, the difference between suppression to $T$ and $T L$ should have developed more rapidly in Group $U$ than in Group B. 
The absence of unblocking in this study can, on the other hand, be accounted for in terms of the RescorlaWagner formulation, if it is assumed that Stage 1 training was insufficient to establish asymptotic excitatory strength to $\mathrm{N}$ for either group. However, for Group $\mathrm{U}$, this training was apparently sufficient to prevent conditioning to $\mathrm{L}$ with the reduced shock employed in Stage 2; whereas, for Group B, further conditioning to L was possible and did occur.

In conclusion, although these results do not correspond with the prediction derived above from the Rescorla-Wagner model, they appear more consistent with that formulation than with the alternative attentional account.

\section{REFERENCES}

Kamin, L. J. Predictability, surprise, attention, and conditioning. In B. A. Campbell \& R. M. Church (Eds.), Punishment. New York: Appleton-Century-Crofts, 1969.
Mackintosh, N. J. A theory of attention: Variations in the associability of stimuli with reinforcement. Psychological Review, 1975, 82, 276-298.

Mackintosh, N. J., \& Turner, C. Blocking as a function of novelty of CS and predictability of UCS. Quarterly Journal of Experimental Psychology, 1971, 23, 359-366.

Rescorla, R. A., \& WAgner, A. R. A theory of Pavlovian conditioning: Variations in the effectiveness of reinforcement and nonreinforcement. In A. Black \& W. F. Prokasy (Eds.), Classical conditioning: II Current theory and research. New York: Appleton-Century-Crofts, 1972.

Sutherland, N. S., \& MaCkintosh, N. J. Mechanisms of animal discrimination learning. New York: Academic Press, 1971.

(Received for publication April 12, 1976.) 